\title{
Análisis bibliométrico de la revista RELIME (1997-2011)
}

\author{
Alexander Maz Machado \\ Noelia Jiménez Fanjul \\ Rafael Bracho López \\ Natividad Adamuz Povedano *
}

Artículo recibido:

4 de noviembre de 2013.

Artículo aceptado:

30 de abril de 2014.

\section{Resumen}

Se presenta un análisis de los 15 años de la revista mexicana RELIME (1997-2011), centrándose dicho análisis en la colaboración entre investigadores y países así como en la temática de los artículos publicados. Se halló que la producción de la revista proviene principalmente de México, España y Argentina. Los patrones de coautoría son similares a otras revistas de investigación en Matemática Educativa. Los aspectos más tratados en la revista son los procesos cognitivos así como las contribuciones teóricas y filosóficas a la Educación Matemática.

* Los cuatro autores pertenecen a la Universidad de Córdoba, España. ma1mamaa@ uco.es

INVESTIGACIÓN BIBLIOTECOLÓGICA, Vol. 29, Núm.66, mayo/agosto, 2015, México, ISSN: 0187-358X. pp. 91-104 
Palabras clave: Revista RELIME; Educación Matemática; Colaboración; Coautoría científica; Análisis de redes académicas; Bibliometría.

\begin{abstract}
Bibliometric analysis of the RELIME journal (1997-2011) Alexander Maz-Machado, Noelia Jiménez-Fanjul, Natividad Adamuz-Povedano and Rafael Bracho-López

This paper presents an analysis of the degree and nature of collaboration among researchers from diverse countries publishing over a fifteen-year period from 1997 to 2011 in the Mexican journal RELIME. The study also looks at topics of published papers. We found that the research papers published in the journal come largely from Mexico, Spain and Argentina. Moreover, co-authorship patterns are similar to those found in mathematics education journals. The research areas most often addressed in RELIME are cognitive processes, and theoretical and philosophical contributions to mathematics education.
\end{abstract}

Keywords: RELIME Journal; Mathematics Education; Collaboration; Scientific Co-authorship; Academics Network Analysis; Bibliometrics.

\title{
INTRODUCCIÓN
}

工 a construcción y difusión del conocimiento científico es un proceso social en el que de manera coordinada un colectivo de investigadores presenta a la comunidad científica los nuevos hallazgos, las estrategias novedosas o los nuevos planteamientos teóricos de una disciplina. De tal forma que se da origen a redes académicas de carácter social y científico, tanto en colaboración personal como institucional o a nivel de países o regiones geográficas, las que se hacen visibles a través de las revistas especializadas. En este sentido la Revista Latinoamericana de Investigación en Matemática Educativa (RELIME) ocupa un lugar destacado y de suma importancia en el ámbito de la Matemática Educativa (o Educación Matemática) en Latinoamérica.

Desde 1987 se venían realizando, de forma anual, reuniones Centroamericanas y del Caribe sobre Formación de Profesores e Investigación en Ma- 
temática Educativa, en las que se observó que existía en la región un amplio colectivo de investigadores sobre este tema, así como un volumen de investigación que requería de un canal de difusión más amplio y formal. Todo esto llevó a constituir en 1996 el Comité Latinoamericano de Matemática Educativa (CLAME). Luego, en el año 1997 se funda la revista RELIME para servir como órgano de publicación oficial del CLAME. El objetivo era promover y fomentar la escritura de artículos de investigación de alta calidad en la región. Como indica Cantoral (2011), la revista pasa por tres etapas: los inicios o nacimiento (un par de años), la maduración y la consolidación e internacionalización, que es la etapa actual. Para el futuro parece que el camino debería ser la consolidación mediante el impacto y repercusión en la investigación internacional de la revista.

Esta iniciativa de la revista RELIME de trascender al campo internacional fue pionera en Latinoamérica y dio inicio a su recorrido con un solo artículo publicado en aquel primer número del volumen 0, del año 1997, que culmina hoy con la celebración de sus 15 años de edición ininterrumpida con su inclusión en las bases de datos internacionales más prestigiosas (SSCI de la WoS, Latindex, SciELO, Redalyc, Dialnet, ZDM y ERIH), estrenando además factor de impacto (FI) en el Journal Citation Reports (JCR) Social Sciences Edition: 0.083. Para reconocer la dimensión y trascendencia internacional de este hecho es conveniente recordar que en el JCR del año 2010 solamente había tres revistas específicas de Educación Matemática (Cantoral, 2010) y RELIME era la única editada en idioma español, aunque aceptaba artículos en inglés, francés y portugués. Por esta razón consideramos pertinente y necesario hacer un balance de la revista, desde su origen hasta obtener FI, mediante indicadores objetivos como los que brinda la bibliometría. Este FI si bien es importante para la revista debe tomarse con precauciones, puesto que entre los muchos aspectos que los investigadores cuestionan de este indicador (Bordons, Fernández y Gómez, 2002) está el hecho de que sólo se consideran las citas recibidas por las revistas fuentes de las bases de datos SCI y SSCI, sabiéndose que existe en ellas un marcado sesgo negativo hacia las revistas de habla no inglesa (Gámez, 2011).

El objetivo del presente estudio es caracterizar la producción científica de la revista RELIME, desde su creación hasta el año 2011, mediante un análisis bibliométrico, con el propósito de estudiar dicha producción así como la colaboración científica entre los investigadores y países. A nivel internacional es frecuente el estudio de las revistas especializadas en todas las áreas científicas (Nebelong-Bonnevie y Faber-Frandsen, 2006; Thanuskodi, 2010; Peñaranda, Quiñones y López, 2005) porque permite detectar frentes de investigación, tendencias y patrones de publicación. La Educación Matemática 
no es ajena a esta tendencia y prueba de ello son los recientes estudios publicados (Maz-Machado et al., 2009; Bracho-López et al., 2012; Maz-Machado et al., 2011).

\section{MÉTodo}

Se han considerado todos los artículos científicos publicados por RELIME, descartando así los editoriales, a lo largo del periodo 1997-2011. Se ha analizado desde el número 1 del volumen 0 hasta el número 3 del volumen 14 . Se accedió a todos los números publicados desde la página web de la revista. La información bibliográfica de los registros ha sido introducida manualmente en una base de datos ad boc de tipo relacional. A continuación se ha llevado a cabo un proceso de normalización de las distintas variantes en las firmas de un mismo autor, así como la asignación del sexo para conocer la participación por género. Algunas de las variantes en las firmas surgen porque en ocasiones los autores firman con un solo apellido y en otras con dos, lo mismo sucede en el caso de los nombres compuestos.

Los datos fueron representados por una matriz de afiliación en la que los valores de cada una de las celdas $i j X$ pueden ser 1 o 0 en función de si se ha firmado o no como autor. La matriz de afiliación refleja las relaciones entre los autores en función del número de coautorías. Este diseño metodológico se ha aplicado en otras investigaciones bibliométricas centradas en la Educación Matemática, por lo que consideramos que tiene un buen grado de validez y fiabilidad (Bracho-López et al., 2012).

Se tomaron como variables de investigación las siguientes:

a) Año de publicación.

b) Nombres y apellidos de los autores.

c) Número de firmas.

d) Filiación institucional de los autores.

e) País donde trabaja cada uno de los autores firmantes.

f) Género de los autores.

g) Temática del artículo.

Para determinar la variable temática se recurrió a la categorización que realiza la base de datos MathEduc, la cual es ampliamente conocida y aceptada por la comunidad científica internacional (Ruffer-Henn y Wegne, 2010).

La colaboración científica de los investigadores se ha determinado a partir de dos indicadores, índice de coautoría (IC) (Lawani, 1980) y grado de co- 
laboración (GC) (Subramanyam, 1983). Así, para una colección K de artículos publicados en una revista, estos indicadores quedan definidos como:

$$
I C=\frac{\sum_{j=1}^{A} j f_{j}}{N} \text { y } \quad G C=1-\frac{f_{1}}{N}
$$

Donde $0 \leq \mathrm{GC} \leq 1$

$f_{1}=$ Número de artículos con un solo autor en una colección $\mathrm{K}$.

$\mathrm{N}=$ Número total de artículos en $\mathrm{K}$.

$\mathrm{A}=$ Número total de firmas en una colección $\mathrm{K}$.

Se identificaron los grupos de investigación o redes de autores que han publicado en la revista, para lo que se han cuantificado todas las coautorías de los artículos, considerándose la existencia de un grupo cuando éste está integrado al menos por cuatro investigadores, siguiendo un proceso semejante al utilizado por Lima (2011). Para la representación de la red global de los grupos de investigación se utilizó el algoritmo Kamada-Kawai (Kamada y Kawai, 1988). También se identifica la red de colaboración internacional en la disciplina. Para construir las redes se ha utilizado el programa Pajek (Batagelj y Mrvar, 2001).

\section{Resultados}

Se han analizado 182 artículos en los que han participado 233 autores diferentes, de los cuales 107 son hombres y 126 mujeres. Estos autores son responsables de 329 firmas, de las que 168 (51.06 \%) corresponden a hombres y $161(48.94 \%)$ a mujeres, lo que indica que no hay sesgo de género en la autoría de artículos en RELIME, sino una representación casi paritaria (Tabla I).

Tabla I. Distribución anual del número de artículos y de firmas en RELIME desglosada por sexos

\begin{tabular}{|c|c|c|c|c|c|c|}
\hline \multirow[t]{2}{*}{ Año } & \multirow[t]{2}{*}{ Núm. artículos } & \multicolumn{4}{|c|}{ Número de firmas } & \multirow{2}{*}{$\begin{array}{c}\text { Total } \\
\text { de firmas }\end{array}$} \\
\hline & & $\mathrm{H}$ & $\%$ & $M$ & $\%$ & \\
\hline 1997 & 1 & 0 & 0 & 1 & 100 & 1 \\
\hline 1998 & 4 & 2 & 50 & 2 & 50 & 4 \\
\hline 1999 & 8 & 5 & 50 & 5 & 50 & 10 \\
\hline 2000 & 15 & 14 & 53.85 & 12 & 46.15 & 26 \\
\hline 2001 & 11 & 7 & 41.18 & 10 & 58.82 & 17 \\
\hline 2002 & 11 & 12 & 63.16 & 7 & 36.84 & 19 \\
\hline 2003 & 10 & 9 & 47.37 & 10 & 52.63 & 19 \\
\hline 2004 & 11 & 8 & 53.33 & 7 & 46.67 & 15 \\
\hline
\end{tabular}




\begin{tabular}{|c|c|c|c|c|c|c|}
\hline 2005 & 17 & 8 & 40 & 12 & 60 & 20 \\
\hline 2006 & 31 & 34 & 54.84 & 28 & 45.16 & 62 \\
\hline 2007 & 15 & 16 & 55.17 & 13 & 44.83 & 29 \\
\hline 2008 & 12 & 13 & 46.43 & 15 & 53.57 & 28 \\
\hline 2009 & 12 & 11 & 40.74 & 16 & 59.26 & 27 \\
\hline 2010 & 12 & 11 & 44 & 14 & 56 & 25 \\
\hline 2011 & 12 & 18 & 66.67 & 9 & 33.33 & 27 \\
\hline Total & 182 & 168 & & 161 & & 329 \\
\hline
\end{tabular}

Podemos ver en la Tabla II que son México, España y Argentina los países que aportan más de la mitad de los artículos, si bien cada uno de los dos primeros duplica la producción argentina. En esta misma tabla vemos que el $21 \%$ de los países contribuyen con un único artículo. Lotka (1926) señala que los autores con 10 o más publicaciones en un campo determinado deben ser considerados como grandes productores. Si realizamos una analogía entre los autores y los países de los autores podríamos afirmar que México, España, Argentina e Italia son grandes productores $(n \geq 10)$ en términos de Lotka.

Tabla Il. Número de artículos publicados por país

\begin{tabular}{|l|c|c|l|c|c|}
\hline \multicolumn{1}{|c|}{ País } & Núm. artículos & $\%$ sobre 182 & \multicolumn{1}{c|}{ País } & Núm. artículos & $\%$ sobre 182 \\
\hline México & 56 & $30.77 \%$ & Francia & 5 & $2.75 \%$ \\
\hline España & 44 & $24.18 \%$ & Portugal & 4 & $2.20 \%$ \\
\hline Argentina & 22 & $12.09 \%$ & Chile & 3 & $1.65 \%$ \\
\hline Italia & 12 & $6.59 \%$ & Alemania & 2 & $1.10 \%$ \\
\hline Brasil & 10 & $5.49 \%$ & Perú & 2 & $1.10 \%$ \\
\hline USA & 9 & $4.95 \%$ & Dinamarca & 1 & $0.55 \%$ \\
\hline Canadá & 7 & $3.85 \%$ & Grecia & 1 & $0.55 \%$ \\
\hline Colombia & 7 & $3.85 \%$ & Inglaterra & 1 & $0.55 \%$ \\
\hline Venezuela & 7 & $3.85 \%$ & Sudáfrica & 1 & $0.55 \%$ \\
\hline Cuba & 5 & $2.75 \%$ & & & \\
\hline
\end{tabular}

Se puede observar que el total de artículos de esta tabla (199) es algo superior al número real de artículos incluidos en el estudio (182), esto es debido a que en un mismo artículo pueden colaborar países distintos. Precisamente es en esta colaboración internacional en lo que a continuación nos vamos a centrar.

En la Figura 1 podemos ver de forma más clara cómo se da la colaboración entre los distintos países en la producción de artículos científicos en Educación Matemática. Cada país está representado por un nodo cuya área es proporcional al número de artículos que aporta respecto del total. Las líneas enlazan países que han colaborado en la producción de artículos, sien- 
do su grosor directamente proporcional al número de artículos en los que han colaborado. Así vemos que hay países como Estados Unidos, Canadá, Cuba, Alemania, entre otros, que han aportado artículos sin colaborar con otros.

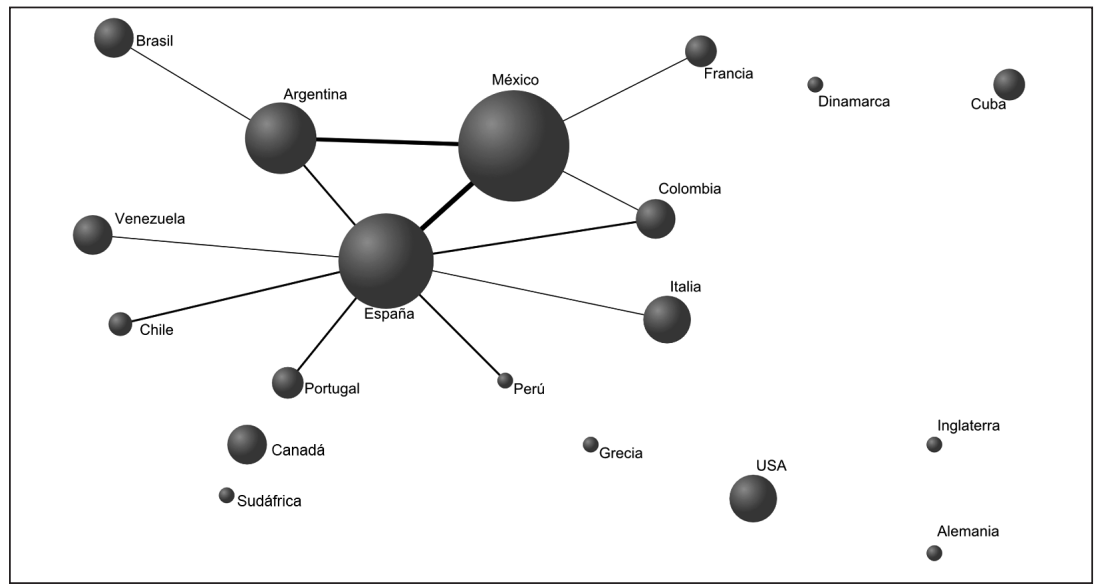

Figura 1. Red de colaboración internacional

Los vínculos o lazos de colaboración más fuertes son México con España y México con Argentina. Observamos una componente de la red de colaboración de tipo estrellado y algunos nodos aislados. Existen dos nodos centrales de esta componente, a saber, México y España, que actúan como enlaces principales de la misma, si bien cabe destacar a México como el mayor productor. Esta componente estrellada está integrada casi en su totalidad por países iberoamericanos, siendo Francia e Italia los dos únicos países no iberoamericanos. Destacamos que, curiosamente y pese a ser Francia un país europeo, encuentra su enlace a través de México y no a través de España, como ocurre con Italia y Portugal. Otro aspecto reseñable es que México está directamente vinculado sólo con dos países latinoamericanos, mientras que España lo está con cinco, lo cual puede deberse a los vínculos que se establecen cuando los investigadores latinoamericanos cursan sus estudios de doctorado en España.

Tras ver los artículos producidos por cada país y la red de colaboración internacional, el paso siguiente es analizar las firmas que aporta cada país y detectar qué relación guarda con el número de artículos de cada uno. Los 182 artículos tienen un total de 329 firmas, lo que supone un promedio de 1.81 firmas por artículo (índice de coautoría), lo que denota la escasa colaboración entre autores. Este índice es semejante al obtenido para otras revistas 
latinoamericanas de Educación Matemática: Enseñanza de las Ciencias (1.84) y Suma (1.74). Estos índices empiezan a vislumbrar un patrón en la coautoría iberoamericana de la disciplina (Maz-Machado et al., 2009).

México, que aporta el $30.77 \%$ de los artículos aproximadamente, sólo es responsable del $26.44 \%$ de las firmas. En cambio España, que es responsable del $24.18 \%$ de las firmas contribuye sólo en el $25.53 \%$ de los artículos. Los países que más amortizan su participación en la producción de artículos son Grecia, Estados Unidos y Brasil, duplicando (y triplicando en algunos casos) el número de firmas en relación al de artículos publicados (Tabla III).

Tabla III. Relación entre el número de firmas y el número de artículos por país

\begin{tabular}{|l|c|c|c|c|c|}
\hline \multicolumn{1}{|c|}{ País } & Núm. artículos A & \% sobre 182 & Núm. firmas B & $\%$ sobre 329 & B/A \\
\hline Grecia & 1 & $0.55 \%$ & 3 & $0.91 \%$ & 3.00 \\
\hline USA & 9 & $4.95 \%$ & 19 & $5.78 \%$ & 2.11 \\
\hline Brasil & 10 & $5.49 \%$ & 21 & $6.38 \%$ & 2.10 \\
\hline Colombia & 7 & $3.85 \%$ & 14 & $4.26 \%$ & 2.00 \\
\hline Portugal & 4 & $2.20 \%$ & 8 & $2.43 \%$ & 2.00 \\
\hline Inglaterra & 1 & $0.55 \%$ & 2 & $0.61 \%$ & 2.00 \\
\hline España & 44 & $24.18 \%$ & 84 & $25.53 \%$ & 1.91 \\
\hline Argentina & 22 & $12.09 \%$ & 39 & $11.85 \%$ & 1.77 \\
\hline México & 56 & $30.77 \%$ & 87 & $26.44 \%$ & 1.55 \\
\hline Cuba & 5 & $2.75 \%$ & 7 & $2.13 \%$ & 1.40 \\
\hline Chile & 3 & $1.65 \%$ & 4 & $1.22 \%$ & 1.33 \\
\hline Canadá & 7 & $3.85 \%$ & 9 & $2.74 \%$ & 1.29 \\
\hline Venezuela & 7 & $3.85 \%$ & 8 & $2.43 \%$ & 1.14 \\
\hline Italia & 12 & $6.59 \%$ & 13 & $3.95 \%$ & 1.08 \\
\hline Francia & 5 & $2.75 \%$ & 5 & $1.52 \%$ & 1.00 \\
\hline Alemania & 2 & $1.10 \%$ & 2 & $0.61 \%$ & 1.00 \\
\hline Perú & 2 & $1.10 \%$ & 2 & $0.61 \%$ & 1.00 \\
\hline Dinamarca & 1 & $0.55 \%$ & 1 & $0.30 \%$ & 1.00 \\
\hline Sudáfrica & 1 & $0.55 \%$ & 1 & $0.30 \%$ & 1.00 \\
\hline
\end{tabular}

Aproximadamente el $44 \%$ de los artículos está firmado únicamente por un autor, siendo el grado de colaboración (GC) de 0.5604, lo que indica que prácticamente el número de artículos escritos en colaboración y los escritos de manera aislada es el mismo, mientras que el $4.4 \%$ de los artículos están firmados por cuatro o más autores. El artículo con mayor número de autores tiene seis firmas. El GC de RELIME es muy cercano al 0.61 hallado para un conjunto de cuatro revistas de Educación Matemática en JCR (Jiménez-Fanjul, Maz-Machado y Bracho-López, 2013).

La colaboración en el periodo estudiado para la revista RELIME queda reflejada de manera más clara al realizar y visualizar de forma gráfica la red 
de colaboración entre autores (Figura 2). El tamaño del nodo representa el número de artículos de cada autor.

De la Figura 2 destacamos que existen componentes de la red más importantes en cuanto a volumen de producción y número de integrantes de las mismas, aunque existen numerosos componentes pequeños $(n<4)$ e incluso autores aislados (no mostrados en la figura). Las principales componentes de la red son de tipo estrellado, con uno o dos autores que lideran y constituyen el enlace entre los autores.

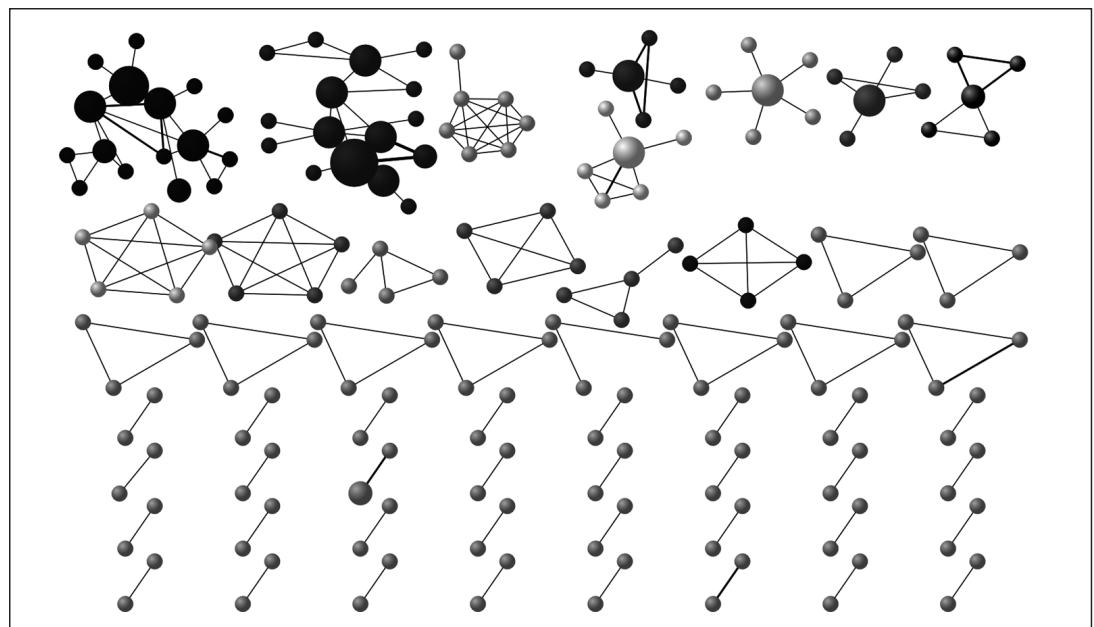

Figura 2. Red de coautoría (total)

Realizando una serie de reducciones nos quedamos con las principales componentes de la red de coautoría en las que al menos intervienen 4 autores (Figuras 3 y 4, p. siguiente). El grosor y color de la línea se corresponde con el número de colaboraciones entre los autores.

En la Figura 3 se observa que la mayor componente de la red de colaboración está integrada en su gran mayoría por investigadores mexicanos, entre los que destacan los de la Universidad Autónoma de Guerrero y el CINVESTAV IPN. Esta colaboración se da alrededor de Rosa María Farfán, Ricardo Cantoral, Gustavo Martínez y Crisólogo Dolores, que son los principales autores de esta componente de tipo estrellado y le dan una cohesión que, pese a incorporar a una investigadora de Argentina y otro de Canadá, es fundamentalmente local.

La segunda componente en tamaño de la red es española e italiana con una presencia mexicana y otra venezolana. El núcleo de colaboración está alrededor de Juan D. Godino y Ángel Contreras. En ella se observa que Bruno D’Amore, pese a ser el autor más productivo (esto es, con más artículos 


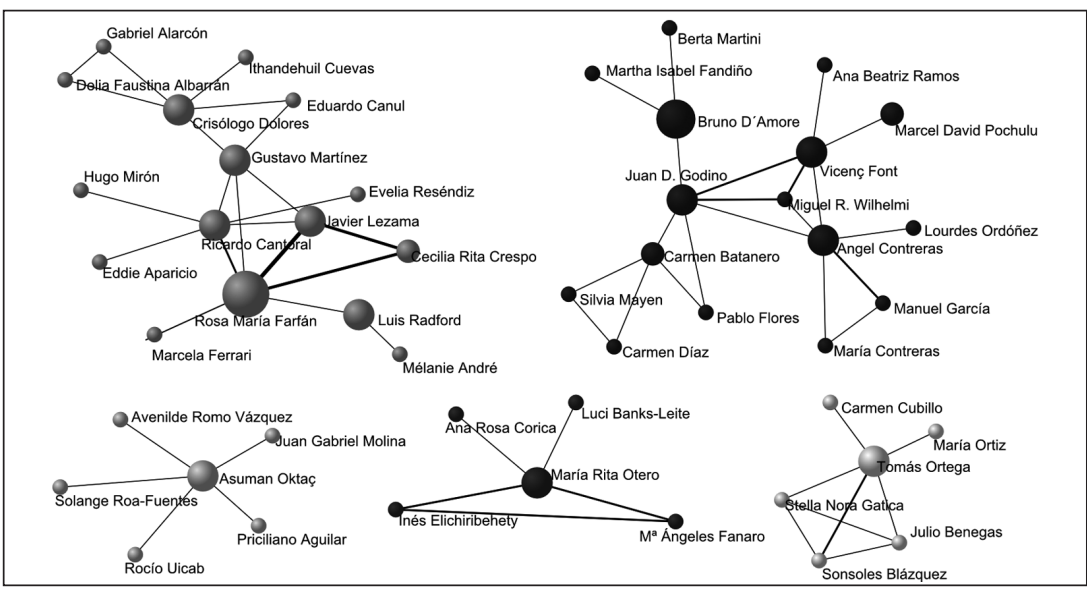

Figura 3. Principales componentes de la red de coautoría (podada)

publicados) no genera a su alrededor una gran conexión de colaboración, lo que indica que firma de manera individual la mayoría de sus artículos.

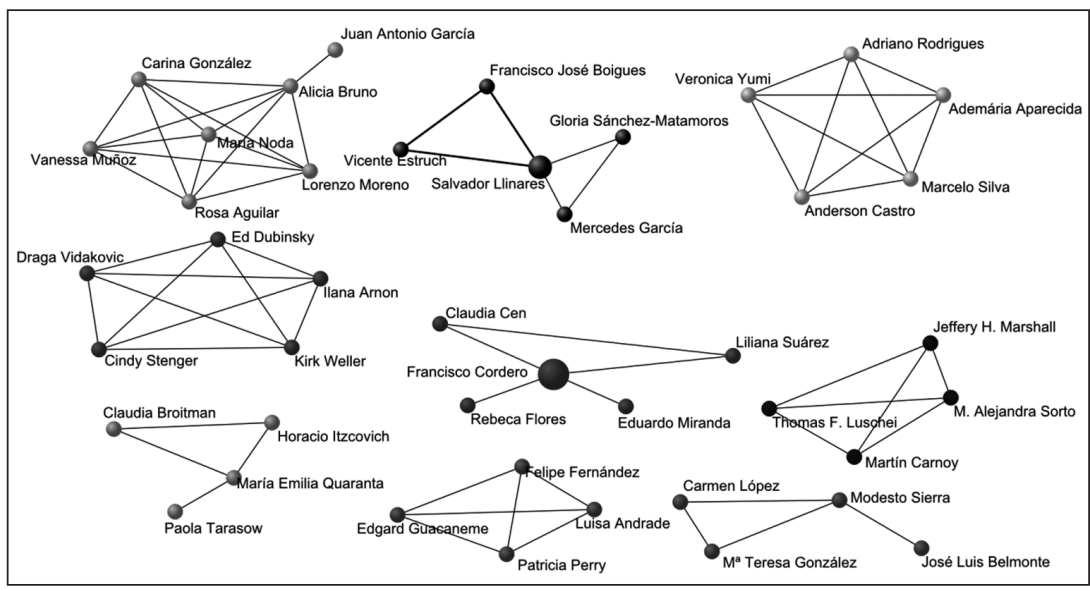

Figura 4. Componentes secundarias de la red de coautoría (podada)

El resto de las componentes importantes identificadas de la red generalmente no son componentes de tipo no estrellado (Figura 4), no destacando ningún autor especialmente como líder y colaborando todos de forma similar. Apreciamos que la mayor componente de éstas es únicamente española y más concretamente de la Universidad de la Laguna.

En la base de datos MatbEduc (http://www.zentralblatt-math.org/mathe duc/), que es específica de Educación Matemática, hay indexados y clasifica- 
dos solamente 135 artículos de la revista, correspondientes a un periodo entre el año 2001 y 2011. Este análisis corresponde a una muestra de los artículos de nuestro periodo de estudio que suponen un $74.17 \%$.

Un análisis temático, según la clasificación temática de la base de datos MathEduc, señala que el $38 \%$ de las investigaciones publicadas en RELIME no tratan sobre un nivel educativo específico, mientras que aquellas relacionadas con aspectos de la Educación Matemática específicos, tanto en la Universidad como en los últimos años de la secundaria, alcanzan juntas el mismo porcentaje (Figura 5). Es llamativo el hecho de que no se publiquen investigaciones sobre la Educación Matemática en la etapa infantil. De igual manera, las investigaciones relacionadas con la formación del profesorado son mínimas.

Un aspecto importante al analizar una revista científica es identificar cuáles son las tendencias temáticas dentro de su área de conocimiento, esto determina los ámbitos o agendas de investigación. Por tal razón y a partir de la clasificación temática que realiza MatbEduc se obtiene que los "Procesos cognitivos. Teorías de aprendizaje" y las "Contribuciones filosóficas y teóricas (Educación Matemática)" son los temas más tratados en la revista (Tabla IV). Las primeras diez categorías temáticas representan un $61.89 \%$ del total, siendo evidente el peso de las categorías D: Educación e instrucción en matemáticas y C: Psicología de la Educación Matemática. Investigación en Educación Matemática. De forma global estas dos temáticas son el $50 \%$ del total. La suma de los artículos por categoría es mayor que el número de artículos publicados en RELIME porque algunos pueden estar en más de una categoría.

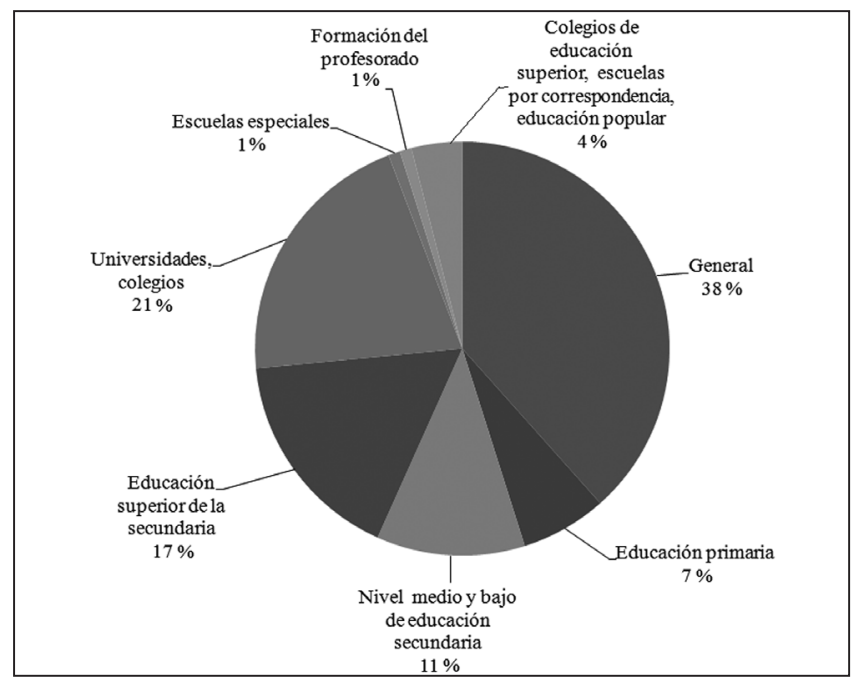

Figura 5. Nivel educativo sobre el que se investiga y publica en RELIME 
Tabla IV. Clasificación temática de los artículos de RELIME según MathEduc

\begin{tabular}{|l|c|c|}
\hline \multicolumn{1}{|c|}{ Clasificación temática } & Núm. & $\%$ \\
\hline C30: Cognitive processes. Learning theories & 49 & 14.94 \\
\hline D20: Philosophical and theoretical contributions (math didactics) & 49 & 14.94 \\
\hline C70: Teaching-learning-processes & 19 & 5.79 \\
\hline I20: Mappings and functions & 19 & 5.79 \\
\hline C60: Sociological aspects of learning & 15 & 4.57 \\
\hline D30: Objectives and goals & 12 & 3.66 \\
\hline D40: Teaching methods and classroom techniques & 12 & 3.66 \\
\hline E20: Philosophy and mathematics & 10 & 3.05 \\
\hline A30: History of mathematics and mathematics education & 9 & 2.74 \\
\hline B50: Teacher education & 9 & 2.74 \\
\hline
\end{tabular}

\section{Conclusiones}

Comparando el número de autores y autoras se concluye que las aportaciones científicas de la revista RELIME no evidencian sesgo debido al género.

A lo largo de sus 15 años la revista ha publicado un número variable de artículos por año, teniendo el mínimo en el primer año (1) y un máximo en el 2006 (31), pero en los últimos cuatro años se ha estabilizado en 12 por año.

Algo más del $42 \%$ de los países cuyos autores han publicado en RELIME lo han hecho de manera aislada, sin colaborar internacionalmente. Esto es indicio de un localismo académico, algo que va en contra de las actuales tendencias de colaboración global y los equipos de investigación de carácter supranacionales. La colaboración que se da a nivel internacional es ocasional, siendo México y España los países principales de dicha red internacional.

Las campos temáticos más estudiados por las contribuciones realizadas en la revista RELIME son los "Procesos cognitivos. Teorías de aprendizaje" y las "Contribuciones filosóficas y teóricas (Educación Matemática)" con casi un $30 \%$ del total de artículos. Esto las señala como las temáticas que copan las agendas de investigación de los países iberoamericanos.

El $15 \%$ de los países aportan más de la mitad del total de artículos, esto indica que la revista está enfocada a países iberoamericanos, siendo los más productivos México, España y Argentina. Estos datos revelan que el propósito inicial de RELIME de ser el mecanismo de difusión de la investigación en Matemática Educativa que se realiza en la región se ha conseguido parcialmente, puesto que sólo nueve países de los 23 iberoamericanos han publicado en la revista. De este hecho no es responsable la propia revista sino que es la consecuencia de los diferentes estados de madurez y desarrollo de la disciplina en la región. 
Como el origen de RELIME obedeció a ser el medio de difusión del CLAME, la inclusión en su título de "Revista Latinoamericana" respondía a las expectativas planteadas por la comunidad investigadora de esa región; sin embargo, el $14.83 \%$ de los artículos publicados provienen de fuera de Latinoamérica. Con la inclusión de la revista en diversas bases de datos e índices internacionales se ha generado un efecto llamada de autores de diversas regiones del mundo y esto irá en crecimiento según aumente su factor de impacto. Por lo tanto, parece razonable que el Consejo Editor de RELIME reflexione acerca de si el título de la revista refleja la procedencia de la autoría de los artículos que publica. Si bien el prestigio que ha adquirido en el campo de la Educación Matemática sería un aspecto de peso a considerar sobre dicho cambio.

Como tarea pendiente para el futuro queda analizar el impacto internacional que la revista ha podido adquirir tras su ingreso en el JCR, en terminos de citación, para poder así compararla con otras revistas de mayor tradición y prestigio dentro de su área de conocimiento.

\section{REFERENCIAS BIBLIOGRÁFICAS}

Batagelj, V. y Mrvar, A. (2001), Pajeck. Program for Large Network Analysis, Slovenia: University of Ljubljana.

Bordons, M.; Fernández, M. T. y Gómez, I. (2002), “Advantages and limitations in the use of impact factor measures for the assessment of research performance in a peripheral country", en Scientometrics, 53 (2), pp. 195-206.

Bracho-López, R.; Maz-Machado, A.; Gutiérrez-Arenas, M.; Torralbo-Rodríguez, M.; Jiménez-Fanjul, N. y Adamuz-Povedano, N. (2012), "La investigación en Educación Matemática a través de las publicaciones científicas españolas”, en Revista Española de Documentación Científica, 35 (2), pp. 262-280. Disponible en: http://re dc.revistas.csic.es/index.php/redc/article/view/736

Cantoral, R. (2010), "Finalmente... TROIS", en RELIME, 13 (1), pp. 5-6. Disponible en: http://www.clame.org.mx/relime/201000a.html - (2011), "La escuela latinoamericana de matemática educativa", en RELIME, 14 (1), pp. 5-8. Disponible en: http://www.clame.org. $\mathrm{mx} / \mathrm{relime} / 201100 \mathrm{a} \cdot \mathrm{html}$

Gámez, A. M. (2011), "Relevancia de las revistas españolas incluidas en las bases de datos del Institute for Scientific Information medida a tráves del factor de impacto renormalizado", en Investigación Bibliotecológica, 25 (55), pp. 67-70. 
Jiménez-Fanjul, N.; Maz-Machado, A. y Bracho-López, R. (2013), "Bibliometric analysis of the mathematics education journals in the SSCI", en International Journal of Research in Social Sciences, 2 (3), pp. 26-32.

Kamada, T. y Kawai, S. (1988), "An Algorithm for Drawing General Undirected Graphs”, en Information Processing Letters, 31, pp. 715.

Lawani, S. M. (1980), Quality, collaboration and citation in cancer research: A 268 bibliometric study, Ph.d. Dissertation, Florida State University.

Lima, M. Y. (2011), "Coautoria na produção científica do PPGGeo/ UFRGS: uma análise de redes sociais”, en Ciência da Informação, 40 (1), pp. 38-51.

Lotka, A. J. (1926), "The frequency distribution of scientific productivity", en Journal of the Washington Academy of Science, 16 (12), pp. 317-323.

Maz-Machado, A.; Torralbo, M.; Vallejo, M.; Fernández-Cano, A. y Rico, L. (2009), "La Educación Matemática en la revista Enseñanza de las Ciencias: 1983-2006”, en Enseñanza de las Ciencias, 27 (2), pp. 185-194.

_-; Bracho-López, R.; Torralbo-Rodríguez, M.; Gutiérrez-Arenas, M. P. y Hidalgo-Ariza, M. D. (2011), "La investigación en Educación Matemática en España: los simposios de la SEIEM”, en PNA, 5 (4), pp. 163-184. Disponible en: http://www.pna.es/Numeros2/ Volumen5Numero4.html

Nebelong-Bonnevie, E. y Faber-Frandsen, T. (2006), "Journal citation identity and journal citation image: a portrait of the Journal of Documentation", en Journal of Documentation, 62 (1), pp. 30-57.

Peñaranda, M.; Quiñones, E. y López, J. J. (2005), "Veinte años de Anales de Psicología: una revista con raíces académicas (19842004)”, en Anales de Psicología, 21 (2), pp. 181-198.

Ruffer-Henn, B. y Wegne, B. (2010), "MathEduc - the reference database for teaching and learning mathematic", en EMS Newsletter, 77, pp. 55-56.

Subramanyam, K. (1983), "Bibliometric studies of research in collaboration: A review", en Journal of Information Science, 6, p. 37.

Thanuskodi, S. (2010), "Journal of Social Sciences: A Bibliometric Study”, en Journal of Social Sciences, 24 (2), pp. 77-80. 